\title{
El Balance Social y las relaciones entre los Objetivos de Desarrollo Sostenible y los Principios Cooperativos mediante un Análisis de Redes Sociales
}

\author{
Miguel Ángel Alarcón Conde \\ Juan Fernando Álvarez Rodríguez
}

RESUMEN: Los Principios Cooperativos, como hilos conductores de la gestión cooperativa, estipulan la necesidad de realizar acciones en beneficio de la comunidad, formar a sus asociados, cooperar entre cooperativas y propiciar acciones para la preservación del medioambiente, entre otros. Esto conlleva a relacionar las prácticas de las cooperativas como contribuciones a los Objetivos de Desarrollo Sostenible (ODS, en lo sucesivo). Por ello, a partir de un análisis de la relación entre Principios Cooperativos y las metas de los ODS se busca una lectura más depurada de estos aspectos. Se utiliza la metodología Delphi, entre 16 líderes del sector cooperativo colombiano, para identificar relaciones entre aquellos principios y los ODS y las relaciones resultantes se analizan a partir de teoría de grafos y el Análisis de Redes Sociales. Los resultados resaltan que la especificidad económica de las prácticas de algunas cooperativas en Colombia devela, luego de verificar la relación entre principios, prácticas y productos esperados, el grado de responsabilidad de las cooperativas con su entorno dados sus hábitos de No Prioridad del Ánimo de Lucro Personalista y en particular sus rutinas de emisión de Transferencias Sociales en Especie (TSE, en adelante), que en definitiva son las prácticas que estas empresas desarrollan en sintonía con los ODS. Estas prácticas podrían reportarse en el Balance Social como una de las herramientas más contundentes para demostrar el aporte de las cooperativas a los ODS.Con estos hallazgos se plantea, como propuesta final, fortalecer los vínculos instrumentales con fundamentos teóricos del institucionalismo económico contemporáneo y las nociones aplicadas de Balance Social, con el fin de contribuir a seguir revelando, en la teoría y en la práctica, el aporte de las cooperativas a los ODS.

PALABRAS CLAVE: Objetivos de Desarrollo Sostenible, Principios Cooperativos, Balance Social, Análisis de Redes Sociales, Transferencias sociales en especie.

CLAVES ECONLIT: B52, 015, 019, P13, Q01, Z13.

Cómo citar este artículo / How to cite this article: ALARCÓN, M.A. \& ÁLVAREZ, J.F. (2020): "El Balance Social y las relaciones entre los Objetivos de Desarrollo Sostenible y los Principios Cooperativos mediante un Análisis de Redes Sociales", CIRIEC-España, Revista de Economía Pública, Social y Cooperativa, 99, 57-87. DOI: 107203/CIRIEC-E.99.14322.

Correspondencia: Miguel Ángel Alarcón Conde, miguelangel.alarcon@uclm.es, Universidad de Castilla-La Mancha; Juan Fernando Álvarez Rodríguez, alvarez_juan@javeriana.edu.co, Pontificia Universidad Javeriana. 


\section{EXPANDED ABSTRACT}

\section{The Social Balance and the Relations Between the Sustainable Development Goals and the Cooperative Principles for Colombia under a Social Network Analysis}

\section{Objectives}

This article lays down two strong elements in the search for a consensus between the indicators that best represent the practices of the Social and Solidarity Economy-based economy (SSE, hereinafter) and their contribution to the Sustainable Development Goals (SDGs, hereinafter), 1) sustainability according to Brundtland, and 2) the idea that something must be achieved "beyond GDP" as an integral indicator of progress, always on the economic, social and environmental level

The tendency for accountability upon the intrinsic aspects regarding social profitability tends to make the metrics micro. In fact, the tools based on social balance which are drawn out raise this perspective and leave out the importance of the aggregates, even if they were groups of component units of cooperativism or belonging to the SSE (sectors, communities, territories of varying levels). It should also be borne in mind that if there is no reference or standard, then there is no possible comparability within entities or components of the social economy. This paper proposes an integration between the meso and macro references as benchmarks for placing the micro aspects.

In addition, in the traditional comparability of the Social Economy, the negative externalities generated by SSE entities are usually waived and we find metrics that only add up and do not subtract, contributing to a lack of representation of reality and for scholars from other fields to look down on our progress as they point to it with an excessive idealization.

Therefore, without forgetting the core of the issue, the objective of this work is to delve into the way in which the elements identifying cooperatives and SDGs correlate each other.

\section{The methodological design}

If the Social Balance reports the incidence of cooperative action and with it the social progress achieved, how much do the results coming from cooperatives contribute to the SDGs? This work aims at identifying which constructs relying on economic theories that govern monetary, non-monetary and convertible measurement should be taken into account. To pursue it, a measurement of Release of Resources, Social Surpluses and Merit Goods of Cooperativism. 
Each of these calculations is based on the fact that there is Non-Persistence in Personalist Profit Motive. Furthermore, the Release of Resources, Social Surpluses and Merit Goods stemming from cooperativism make up routines that result, technically speaking, in extended Social Transfers in Kind (STK, hereinafter), but they are on the "expanded way". These determine the differentiating factors of cooperatives (and of other social-economy-based companies) in contrast to capitalist firms. These issues are closely related to the institutionalist view regarding social and cooperative economy.

On the other hand, the graphic expression of a network linking the SDGs to the Cooperative Principles either at a micro level (cooperative) or at a certain level of aggregation (whether sectorial, spatial, by type of cooperative, and/or for each component of the social economies) shapes the graph which may clarify the concepts network as far as centrality and cohesion of the network of relationships is concerned. Thus, if a node has more relationships with the rest and these are even sounder, it will be considered the head in the network. The node centrality can be located on the graph depicting the network based on algorithms available on UCINET 6 and Netdraw. Thus, the transversality of the cooperative principles and SDGs is inferred.

As expressed above and based upon the results of the Delphi method, a group of experts on cooperativism from CIRIEC-Colombia and the Colombian cooperative community evaluate the contribution of cooperativism to the SDGs in a mode 2 data matrix on a Likert scale 1-3,from which it is possible to obtain networks based on the transversality of the cooperative principles by means of common SDGs, or of SDGs under common Cooperative Principles.

In this sense, to approximate the correspondence between the 17 SDGs and the 7 Cooperative Principles, the experts will break down the goals of each of the 17 SDGs and apply a scale to the contribution of the cooperative principles towards them.: 1. Hard, 2. Marginal, 3. Adequate. Then, an average is obtained from the scale of the goals making up each SDG. At the same time, the relationship between the cooperative principles and the SDGs is cross checked so that the correlations offer a valuable network as regards intensity of the contribution between the nodes (Principles and Goals) involved.

For the "Core-Periphery" analysis of the network, that is, of the most intense versus least intense interrelationships, the "hill climbing" algorithm is applied to UCINET 6, which adjusts itself to the ideal division of the nodes using the number of relationships for binary data in an initial split and an eigenvector for the valued data available for the matters, along with several random divisions. This algorithm iterates to fit the initial splits of the nodes and reports the best alignment.

\section{Results}

The Cooperative Principles can be characterized as specific, with a mainly internal repercussion, such as the principles of Open and Voluntary Membership, Democratic Member Control and Autonomy 
and Independence as well as of broad environment. The last principles, such as that of Education, Training, and Information, Concern for Community and Cooperation Among Cooperatives, among others, are protagonists of an additional contribution outside the specific cooperative environment, understood as additional externalities, due to the fact that many SDGs are broken apart into goals to which, almost exclusively, one can contribute through public policies and they are not easily opened to the cooperatives' contributions.

Another network of SDGs-cooperative principles clusters, at its core, the principles concerned with Members' Economic Participation, Equality, Education, Training, and Information, Cooperation Among Cooperatives and Concern for Community, together with the SDGs Sustainable Cities And Communities and Quality Education.

The article highlights that, due to its representation of centrality, "power" is itself aligned with the more central nodes and the principle of Concern for Community, for being the node with the highest concentration to the contribution of the SDGs. Secondly, it outlines the principle of Education, Training, and Information, and so on until the most peripheral principles are reached; but nonetheless important, the relationship of Autonomy and to SDGs is likewise significant for cooperative endeavors and stands out for its capacity to contribute to the accomplishment of any SDG and ostensibly reduces with regard to the first ones.

The representation of the Cooperative Principles of Concern for Community, and of Equality are also highlighted as transversal axes of the remaining cooperative principles and a good set of SDGs. This is what follows when applying the analysis of main components to the matrix of relationships between the cooperative principles and SDGs.

Finally, a clustering is obtained from the cooperative principles (SDGs), which offer a certain taxonomy of possible relationships between the components of both dimensions. Belonging to the same cluster means that properties are shared. Thus, 4 groups of nodes are obtained. Their distinguishing features are the following ones:

1- Strong transversality. This grouping constitutes the strongest cross-cutting relationships and interdependencies between cooperative principles and the SDGs.

2- General collateral transversality. This grouping of relationships is configured on the positive externalities of cooperativism towards the more general environment.

3- Specific collateral transversality. This cluster is configured on positive externalities of cooperativism towards its more specific environment.

4- Implicit transversality. This grouping of relationships supposes potential effects of internalization towards cooperativism as an institution. 


\section{Conclusions}

Once the network analysis is carried out, it is possible to derive a close relationship between the Cooperative Principles and the aims of the SDGs, and as far as the Colombian model is concerned, there is evidence that cooperatives contribute to the fulfillment of the SDGs by virtue of the enhancement of their principles. The questionnaire survey implemented to 16 Colombian cooperatives shows, for instance, that cooperatives generate between 10 and 15 percentage points of STK (expanded) on average to groups that cover diverse stakeholders.

The findings emphasize that principles such as Concern for Community, and Education, Training, and Information, to mention some, are more related to the fulfillment of the SDG goals and, therefore, the practices carried out in response to these principles will tend to generate more transversal contributions. But this does not undermine the importance of the other cooperative principles as they play a part in the organizational environment of multiple actors making up the cooperatives. In this way, the findings reinforce cooperative identity as a core element to direct contributions of cooperatives to the decisions made under Agenda 2030.

\section{Original Value and Limitations}

The work notes that, at this time and because of partiality, it is feasible to reach consensus on micro, meso and macroeconomic indicators to perform comparative and differentiated analyzes regarding each geography aimed at giving a usefulness to Social Balances as an instrument for the construction of Public Policies. Therefore, there are still enormous challenges such as working on tools that allow bundlings between individual social balances, promoting criteria to value the release of resources generated by the differentials that cooperative members obtain for their transactions with their cooperative in relation to market prices, quantifying social surpluses defined as those goods and services that cooperative members get from non-monetary actions and valuing preferred assets.

To optimize the report about the contributions of cooperatives to the SDG goals, it is possible to appeal to the addition of data to the social balance and to meso-economic aggregation tools. Then, once both link to certain "optimal" indicators in the macroeconomic dimension and summarize the economic and social circuit of the activity concerned with SSE entities, particularly that of cooperatives, as a kind of satellite accounts. It would be advisable to focus especially on the calculation of the levels of the Use of Disposable Income Account and Use of Adjusted Disposable Income Account in National Accounts, which are those that concentrate themselves on the STK treatment.

For reasons of time and cost, it has not been possible to extend the number of experts per country to achieve the interrelationship networks between cooperative principles and SDGs for a wide range of countries, which would have provided the comparability component always required in the applied research. Consequently, the Delphi method could have been applied to a survey with the SSE entities' 
stakeholders and not only to cooperatives. With this, it is not possible to generalize conclusions that are only valid in the cultural, territorial and sectoral environment already described and for the theoretical proposal already pointed out.

KEYWORDS: Sustainable Development Goals, Cooperative Principles, Social Balance, Social Networks Analysis, Social transfers in kind.

\section{Introducción}

El 18 de diciembre de 2009, en el marco de la $65^{\circ}$ sesión plenaria de la Asamblea General de las Naciones Unidas, se aprueba la Resolución No. A/RES/64/136: Las cooperativas en el desarrollo social (ONU, 2010). En dicha Resolución se reconoce que las cooperativas promueven la participación de las personas en el desarrollo socioeconómico y tienen potenciales para contribuir al logro de los objetivos globales en torno al desarrollo sostenible.

Por esta singular asociación de intereses entre las Naciones Unidas y el movimiento cooperativo internacional se proclamó el año 2012 como el Año Internacional de las Cooperativas, alentando a los gobiernos a fomentar de manera articulada a las empresas cooperativas en sus territorios y presten especial atención a la contribución de las cooperativas en la aplicación y seguimiento de los principales tratados internacionales en pro de la sostenibilidad planetaria.

De estas iniciativas destacan estudios que señalan una relación positiva entre el fomento de cooperativas y el desarrollo de prácticas para satisfacer los ODS (ONU, 2014: OIT-ACI, 2014, Martí, 2016; Álvarez, Zabala y Orrego, 2016) que apuntan al logro de la sostenibilidad de manera integral.

El problema estriba en que para los ODS se han desarrollado herramientas de seguimiento institucional que reportan los esfuerzos que hacen los Estados para cumplirlos (ONU, 2018), pero no hay un registro de las herramientas para identificar el aporte de organizaciones como las cooperativas que, a pesar de no estar articuladas con el Estado, desarrollan prácticas que contribuyen al cumplimiento de las metas de éstos (OIT-ACl, 2014). De hecho, varias iniciativas logran ser desarrolladas por el accionar de cooperativas en ciertos territorios (CES-España, 2016; Salathé-Beaulieu, et al., 2019) sin que necesariamente se ajusten al cumplimiento de pactos, acuerdos y tratados en los que la percepción es que no son vinculantes. 
Lo anterior, trae como consecuencia que las cooperativas no prioricen en sus agendas estos compromisos y que los obstáculos propios a los que se enfrentan en su cotidianidad puedan pesar preponderantemente en el cálculo de sus resultados. Con ello se produce un fenómeno por medio del cual las organizaciones y los ciudadanos perciben la incidencia de las organizaciones, pero no hay suficiente documentación para demostrarlo (Social Economy Europe, 2013).

Ello hace parte de la invisibilidad de las organizaciones y no contribuye a poner de manifiesto la incidencia de las cooperativas en la construcción de renovadas formas de sostenibilidad empresarial, de dinamización de los territorios y de consolidación de condiciones indispensables para el desarrollo local: democracia, estructuración de mercados a partir de la retención y agregación de valor a la producción y dinamismo cívico (Felber, 2013). Por ello el discurso de la potencialidad de las cooperativas para construir progreso social termina encontrándose con la falta de evidencia documentada (Roelants, 2013).

En consecuencia, es menester desarrollar acercamientos conceptuales y empíricos que apunten a demostrar tales relaciones. Esto se confirma al ser objetivos para la Alianza Cooperativa Internacional ( $\mathrm{ACl}$, en adelante), como para muchos estudiosos de la materia, estudiar en qué prácticas deben sustentarse las cooperativas para lograr la sostenibilidad integral de su entorno general y específico, y ser las organizaciones de mayor crecimiento en el mundo. De tal manera que se trabaje para cerrar las diferencias entre la vocación sostenible de las cooperativas y la demostración efectiva de su potencialidad (Roelants, 2013; Satgar, 2013).

Para profundizar en estas prácticas, el Instituto de Investigación de las Naciones Unidas para el Desarrollo Social (UNRISD), desarrolló junto a la Organización Internacional del Trabajo (OIT) un encuentro internacional durante el mes de junio de 2019 con el título Implementing the Sustainable Development Goals: What Role for Social and Solidarity Economy ? $^{1}$ con el objetivo de comprender el desarrollo de la Economía Social y Solidaria (ESS, en adelante) en diferentes regiones y territorios, examinar el papel de la ESS como un medio de implementación para los ODS en diversos contextos locales e identificar metodologías sólidas y soluciones innovadoras para medir la ESS y sus impactos.

Las principales conclusiones de ese think-tank se orientan a resaltar los aportes de las cooperativas a los ODS relacionándolos con su capacidad de incidir en la planificación territorial o urbana y la provisión de servicios esenciales. También se destacaron estudios a profundidad donde se pusieron de relieve múltiples aportes, pero en particular se los relacionados a igualdad de género, generación de alimentos y agricultura y el desarrollo de iniciativas con un enfoque eco-social que generan transiciones a la sostenibilidad general (y, obviamente, la específica). En cuanto a la medición de estos aportes, se puso de relieve la necesidad de distinguir la identificación de variables propias del éxito en empresas capitalistas como ingresos y estados financieros de los aportes de la ESS que ponen énfasis en la cohesión social, territorial y el empleo (UNRISD, 2019).

1.- http://unsse.org/knowledge-hub/conference-summary/ 
Un avance aún más reciente es el de Salathé-Beaulieu (et. al., 2019), que realiza un estado del arte y compendio de las herramientas, pero sin intentar homogeneizar para el conjunto de entidades componentes de la ESS, que incluyen las aportaciones más significativas sobre "...la teoría del cambio; los flujos de contabilidad social, informes y auditoría; diversas herramientas desarrolladas por el sector cooperativo; el concepto de "utilité sociale" (utilidad social) elaborado en Francia; el marco Sustainable Livelihoods; el método de retorno de la inversión social (SROI); el banco de indicadores IRIS; el sistema de evaluación de impacto desarrollado por B Lab; y los principios propugnados por el Impact Management Project."

En ese entorno de difícil búsqueda de un consenso entre los indicadores que mejor representan las prácticas de las entidades de la ESS y su contribución a los ODS, este artículo sostiene que hay dos elementos de fuerza: 1) la sostenibilidad a lo Brundtland (1987), y 2) la idea de que hay que conseguir algo "más allá del PIB" como indicador integral de progreso, siempre en el plano económico, social y ambiental (Stiglitz, Sen y Fitoussi, 2009). No obstante, la múltiple disponibilidad de técnicas supone, en sí misma, la mayor limitación para la búsqueda de una armonización y homogeneización de indicadores (y de los criterios para encontrarlos y para compararlos), debido a: 1) las limitaciones culturales y territoriales propias de la generación de esas herramientas y técnicas propuestas (Salathé-Beaulieu, et. al., 2019), y 2) a la intención forzosa de contemplar de un análisis abajo-arriba, sin un ajuste fino que tantee alternativas en métricas que son difícilmente aplicables para comparaciones en el tiempo y en el espacio. Es decir, entre espacios económicos y entre entidades o grupos de ellas.

Lo anterior fuerza a que las métricas sean micro. De hecho, las herramientas que se divulgan sobre Balance Social se plantean en esa óptica, y olvidan la importancia de los agregados, aunque fueran éstos simples grupos de unidades componentes del cooperativismo o de la ESS (sectores, comunidades, territorios de diferentes niveles de magnitud). Tampoco debe olvidarse que si no hay una referencia o estándar, entonces no hay comparabilidad.

Además, si esta comparabilidad renuncia a incorporar las externalidades negativas que las entidades de la ESS generan, encontraríamos métricas que sólo suman y no restan, contribuyendo a una falta de representación de la realidad y a que estudiosos de otros ámbitos miren con recelo nuestros avances, por señalarlos con un exceso de idealización de los emprendimientos sociales y solidarios. Bien es cierto que la contribución neta de las ESS viene a intuirse positiva, pero no necesariamente, en la "escala" de Brundtland (1987), ya que sus externalidades positivas económicas, sociales y ambientales vienen a mostrarse en mayor medida que las negativas, pero debe someterse a contraste según casuística. Rigor, razón y querer un mundo mejor no están reñidos si se desanclan prejuicios y se engarzan fundamentos teóricos fuertes que se evidencien una Economía Aplicada de la Economía Social, Sostenible y Solidaria.

Por tanto, el objetivo del presente artículo es profundizar en la relación entre la identidad de las cooperativas y los ODS. Para ello se identifican algunos de los últimos estudios sobre la materia, identificando los elementos centrales de la identidad cooperativa con los ODS y a partir de qué herramien- 
tas podrían reportarse. Seguidamente se analiza a través de qué mecanismo pueden éstos reportarse en las prácticas cooperativas, poniendo de relieve la necesidad de identificar relaciones entre metas y prácticas. A partir de esto se indaga, partiendo de la metodología Delphi obtenido entre estudiosos y líderes del sector cooperativo colombiano, cómo pueden relacionarse Principios Cooperativos y ODS. Las relaciones resultantes se analizan a partir de teoría de grafos y el Análisis de Redes Sociales. Un apartado de conclusiones da cierre al artículo.

\section{Acercamientos conceptuales desde la concep- ción de Balance Social}

El conjunto de los reportes que rindan cuentas del cumplimiento de los Principios Cooperativos, las estrategias desarrolladas para alcanzarlos y los beneficios que obtienen los asociados y demás grupos de interés por el accionar cooperativo, tienen asiento en el Balance Social. De manera que este puede identificar cómo repercuten los esfuerzos empresariales/cooperativos en pro del mejoramiento de la calidad de vida y el bienestar de sus grupos de interés (Álvarez, 2017).

En consecuencia, el Balance Social no es una memoria ni un informe de actividades, sino un balance periódico y comparado de variables estandarizadas y verificables del grado de cumplimiento de la identidad cooperativa, su promesa empresarial y sus aportes al mejoramiento y preservación de la calidad de vida de los grupos de interés en su entorno (Álvarez, 2017). Bajo esta lectura, el Balance Social es una herramienta útil para que las cooperativas demuestren sus aportes a los ODS. Así, ese balance microeconómico y social se encuentra como herramienta para la evidencia del espacio conceptual de Economía Social y Sostenible que ya se notó en el epígrafe introductorio (llustración 1, Alarcón, 2019). 


\section{Ilustración 1. Economía Social Sostenible desde el Institu- cionalismo Económico}

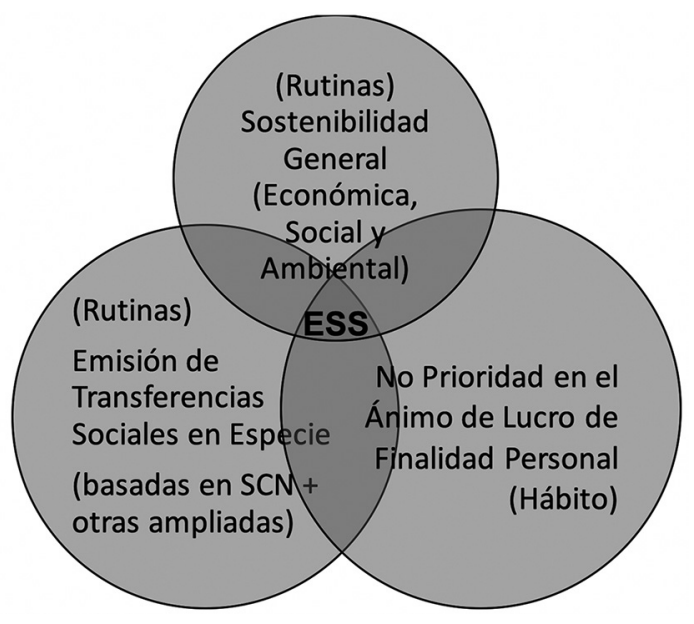

FUENTE: Elaboración propia.

Hay diversos enfoques de reporte del Balance Social. Si su objetivo es medir el grado de cumplimiento de la naturaleza de una cooperativa, la herramienta más usada es el Balance Social Cooperativo desarrollado inicialmente por autoras como Aitziber Mugarra (2001) e instituciones como la ACl, en el que se valorizan acciones concretas por medio de las cuales se cumplen cada uno de los Principios Cooperativos. De estas valoraciones pueden y deben desprenderse también las cuantías que se transfieren a cada uno de los asociados y demás grupos de interés. A esto se convino denominar valor agregado cooperativo. Y, desde este punto de vista, el Balance Social adquiere una dimensión de reporte multistakeholders que acentúa en calcular la diferencia de los bienes y servicios que transfieren las cooperativas a sus asociados, trabajadores, proveedores y comunidad como aproximación a su incidencia.

Otro de los mecanismos para medir el valor agregado proviene de la teoría económica del coste-beneficio, por el que se identifica cuánto cuesta generar un bien o servicio con atributos similares entre firmas capitalistas y cooperativas o cuántos beneficios se pueden reportar a similares costos (Álvarez y Blanco, 2014). Independientemente del supuesto de comparación entre organizaciones, lo fundamental es que se utilizan los precios de mercado como aproximación del bienestar que obtienen los stakeholders. Se trata, en definitiva, de valorar a precios de mercado la liberación de recursos que brindan las cooperativas a sus beneficiarios, cuánto tendrían que pagar en el mercado si no tuvieran fondos sociales que cubren riesgos cotidianos o cuál es el valor de los programas de educación, salud 
y de otros servicios de los que se dispone por estar en una cooperativa y reportarlos como excedentes no contabilizados de la actividad.

También podrían contemplarse Balances Sociales bajo la identificación del cumplimiento de los planes de una organización, siempre que estos respondan a la búsqueda de cumplir el objeto social de ésta y generar bien común en su entorno. Este enfoque tendría sentido si los planes de desarrollo reflejan el cumplimiento de la voluntad de los grupos de interés en el marco de las transacciones que se dan entre la empresa y sus asociados como acto cooperativo (García, 2017). Para Argandoña (2015) el bien común en el entorno hace referencia a la manera de tomar decisiones:

"Fomenta la cooperación, no la confrontación; "da espacio", siempre que sea posible, "al principio de gratuidad" y a la solidaridad; no atribuye la solución de los problemas a las instancias de nivel más alto, sino que implica a todos en su estudio, búsqueda de soluciones e implementación, de acuerdo con el principio de subsidiaridad: da, por tanto, participación a todas las instancias, y principalmente a los directamente afectados por el problema; fomenta la cooperación y el autocontrol; procura la mejora de las instituciones, leyes y regulaciones, etc."

Pero para otros estudiosos esto no sería suficiente, pues lo que debe demostrarse es cuántos beneficios se perciben en una cooperativa cada vez que se invierte una unidad monetaria (Narrillos, 2012). Los beneficios bajo este enfoque estarían basados en las ideas ya maduradas hace más de 30 años por la comisión encargada de realizar Nuestro futuro común (Brundtland, et. al., 1987), como la de contabilizar la sostenibilidad desde un triple resultado: ambiental, económico y social. Pero conviene, también tres décadas después, que pueda considerarse el cooperativismo como institución en vez de ser asociada a una versión de organización individual, aislada y microeconómica, fuera de su conjunto meso-económico. El resultado práctico consistía en derivar la aplicación de Balances Sociales de las instituciones sin ser contextualizados respecto al conjunto institucional al que pertenecen, desvirtuando aspectos como la puesta en común de recursos físicos y de trabajo por parte del genérico "cooperativismo".

Partiendo de la consideración del valor económico, social y ambiental que puede desarrollar una cooperativa, como pueda ser a partir de un informe de sostenibilidad a través de los estándares que sugiere Global Reporting Initiative (su última versión data de 2014, la G4, con algunas actualizaciones recientes que no corresponden a una G5, en 2018 - http://www.globalreporting.org/), u otros que pueden elaborarse por un número importante de consultoras de distintas tonalidades ideológicas, se busca identificar las acciones generadoras de valor y generar certificaciones a partir de estándares que en muchos casos se contemplan en los ODS (Utting, 2018). Pero ¿qué valor comparativo tiene la comparación entre reportes de criterios diferentes y no bajo criterios estrechamente cercanos?

También es posible, en la amplia gama de posibilidades de medición del Balance Social, medir cuál es el aporte al bien común generadas por las transacciones cooperativas. En dicho caso el acen- 
to se colocaría en calcular por los stakeholders los bienes comunes deseables para una sociedad (Felber, 2013). Felber, quien es el autor referente en esta materia, propone valorar a las organizaciones por sus aportes integrales al bien común y no sólo por sus magnitudes financieras. Para ello propone el cálculo de una matriz que cruza los derechos fundamentales y deseados de una economía más humana con los stakeholders que la hacen posible. El resultado: un balance del bien común por medio del cual se pueden hacer rankings de éxito empresarial y de incentivos económicos. La mayoría de las aportaciones pasadas y recientes en el asunto siguen insistiendo en los resultados de la unidad productiva, pero no de la agregación ni de la relativización sobre un estándar con el que hacerlo, mostrándose, solo en ese punto, propuestas incompletas (Bassi y Vicenti, 2015; Retolaza et al., 2015; Etxezarreta, et al., 2018)

No obstante, el reto en el que debe ahondarse en los enfoques mencionados, incluidas las herramientas recopiladas por Salathé-Beaulieu (et. al., 2019), y otros tantos más que existen, es seguir avanzando en la consolidación de un Balance Social como herramienta de agregación y no sólo de orientación al reporte individual de las organizaciones, que podría desvirtuar la idea de fondo: "yo aporto al resultado común, pero me da igual ese resultado común, porque aporto". Además de eso, debería contemplarse la valoración neta, de calado, la de fondo; es decir, no sólo de iniciativas en favor del bien común, sino también reportar aquellas prácticas que impactan negativamente en los resultados del entorno, sea más específico, al propio cooperativismo, o más general. Y, por tanto, tener también en cuenta las externalidades negativas.

Álvarez (2017) destaca que, para abarcar la integralidad del accionar cooperativo, el Balance Social debería contemplar:

- Grado de cumplimiento de la Naturaleza: evidencias de estrategias para el cumplimiento de la naturaleza organizacional cooperativa, manifestadas en acciones e indicadores de cumplimiento de los principios cooperativos,

- Cumplimiento de la función objetivo: evidencia de la satisfacción del bienestar de sus grupos de interés calculada a partir de la maximización de las utilidades netas sociales per cápita de los asociados y el valor transferido a cada grupo de interés,

- Eficiencia administrativa interna: relación de actividades socioeconómicas en el marco del cumplimiento del acto cooperativo.

- Las contribuciones organizacionales a la sostenibilidad.

Como el Balance Social reporta la incidencia del accionar cooperativo y con ello del progreso social que se logra en el ámbito local, la pregunta es ¿cuánto aportan los resultados de las cooperativas a los ODS? La propuesta que se defiende aquí trata de identificar qué constructos ha de tener en cuenta para las mediciones monetarias, no monetarias y convertibles.

En consecuencia, es menester partir de algunas particularidades relacionadas al reporte del Balance social. Dada la polivalencia de actividades que generan las cooperativas para el beneficio de 
sus grupos de interés, podríamos identificar actividades con beneficios monetarios y otras con beneficios no monetarios.

Acercando este argumento a la teoría económica este trabajo propone conceptos susceptibles de visibilizarse en distintos planos. Es decir, se propone la medición sobre:

- Liberación de recursos, como los diferenciales frente a los precios de mercado que obtienen los cooperativistas por sus transacciones con ellas (Álvarez y Blanco, 2014; Alarcón, 2016),

- Excedentes sociales, como aquellos bienes y servicios que obtienen los cooperativistas por las acciones no monetarias (aunque potencialmente convertibles) que se realizan en su organización (Alarcón, 2016, 2019),

- Bienes preferentes o meritorios del cooperativismo: Como aquellos bienes y servicios (educación, salud, seguros) que obtienen los cooperativistas producto de las decisiones democráticas para maximizar su bienestar colectivo, aunque individualmente no estén dispuestos a pagar por ellos (como los beneficios derivados de los fondos sociales) y que terminan expresándose en bienes públicos no estatales.

Cada uno de estos cálculos se basan en que no hay una finalidad personal de lucro en la procura del quehacer empresarial. Por el contrario, la liberación de recursos, excedentes sociales y bienes preferentes del cooperativismo se constituyen en rutinas que terminan siendo, técnicamente, TSE ampliadas $^{2}$, que desde el punto de vista económico determinan los factores diferenciadores de las cooperativas (y otras empresas de la economía social) en contraste respecto a las firmas tradicionales societarias o capitalistas. Aspectos todos relacionados estrechamente con la concepción institucionalista de la Economía Social y Cooperativa (Alarcón, 2016; Alarcón y Álvarez, 2018)

Si bien el término Transferencias Sociales en Especie (TSE) es una partida de la Cuenta de Redistribución de la Renta en Especie ya desde el Sistema de Cuentas Nacionales 1993 de la ONU y del Sistema Europeo de Cuentas Económicas Integradas $1995^{3}$, son el reflejo estadístico de una definición extensa de bienes preferentes contabilizados monetariamente, no ficticios, ya que «...comprenden los bienes y servicios individuales proporcionados a los hogares como transferencias en especie por unidades de las Administraciones Públicas y las ISFLSH (instituciones sin fines de lucro al servicio de los hogares), tanto si se han adquirido en el mercado, como si proceden de la producción no de mercado de las unidades de las Administraciones Públicas y las ISFLSH» (Eurostat, 1996, párrafo 4.104).

2.- Las TSE se emiten por las AAPP y las Instituciones Privadas Sin Fines de Lucro al Servicio de los Hogares por la provisión de bienes y servicios no destinados a la venta, y que reciben los Hogares. Pero la incidencia de las cooperativas en términos de aportes al bien común y el interés general vienen determinadas por ciertas TSE ampliadas. Su cálculo representa no sólo la acción básica cooperativa, que arregla fallos de Estado y de mercado, sino que genera una liberación de recursos públicos para el bien común.

3.- Por tanto, contempladas en el Sistema de Cuentas Nacionales 2008 y SEC 2010 en vigor.

CIRIEC-España, Revista de Economía Pública, Social y Cooperativa ISSN: 0213-8093

N 99/2020, pp. 57-87 
Las TSE Ampliadas, corresponden a ideas que caben en aquel término, pero sus estimaciones no se consideran en los Sistemas de Cuentas Nacionales. Aquí se propone que esas cifras se terminen visibilizando, especialmente para determinar los aportes de las cooperativas a los ODS, que es la institución que las concibe como una de sus rutinas debido al hábito de no ánimo de lucro de finalidad personalista (Alarcón, 2016).

De esa manera, en un Balance Social riguroso de una cooperativa debería presentarse la información de los recursos que se liberan para los beneficiarios de sus transacciones, sus excedentes sociales y los bienes preferentes o meritorios producidos por su accionar. Estas magnitudes serían Transferencias Sociales en Especie ampliadas. y cabrían en los Sistema de Cuentas Nacionales concretando convenciones contables y/o salvedades al criterio general.

Sin embargo, dichas dimensiones siguen siendo particulares y, al no existir un Plan Único de Cuentas de las entidades de la ESS, se hace muy difícil agregar los Balances Sociales de un número significativo de cooperativas para tratar de acercar cuál y cuánto es su aporte agregado al cumplimiento institucional del cooperativismo a los ODS, o su contribución agregada a la Agenda 2030 (anterior, por analogía, Plan 2020). A ello debe sumarse que la realización de múltiples y diferenciadas actividades empresariales hacen compleja la agregación entre entidades. Además, la presentación de un Balance Social es de iniciativa voluntaria, no obligatoria. Por tanto, una cosa es que sea una tarea compleja; otra, que no sea posible.

\section{De los Principios Cooperativos a los ODS. Un análisis de redes}

Una red es una correspondencia entre nodos y enlaces entre estos nodos. Su expresión matemática se denomina grafo. Un grafo $G$ es un par ordenado $G=(N, E)$, donde $N$ es un conjunto de vértices 0 nodos, y $\boldsymbol{E}$ es un conjunto de enlaces, arcos 0 aristas. Es decir, lo que relaciona esos nodos. El grafo es dirigido cuando hay aristas que tienen un origen y un destino. Así, las relaciones entre nodos pueden extenderse a relaciones entre Principios Cooperativos y Objetivos de Desarrollo Sostenible, que se pueden trasladar a una expresión visual que representaría un conjunto de lazos (aristas, nexos, las relaciones) entre los caracteres a representar (nodos, vértices), que son esos Principios y Objetivos.

Así pues, se puede definir una red de Principios Cooperativos-Objetivos de Desarrollo Sostenible, siempre dadas las díadas entre esos dos tipos de nodos y la relación que pueden mantener. La matriz desde la que se construye el grafo es una matriz llamada "de modo 2", también denominada una red de modo 2, en tanto relaciona dos tipos de nodos y no pretende representar las relaciones entre 
Principios Cooperativos o entre ODS por separado. De intentar observar las relaciones entre ODS o entre Principios Cooperativos, se tendrían matrices "de modo 1", que ofrecerían el grafo de una red (modo 1) convencional.

Ahora bien, es posible obtener redes de modo 1 a partir de matrices de modo 2, en tanto es posible definir que, si un Principio Cooperativo comparte cierto ODS, entonces los Principios Cooperativos están relacionados, debido a que tienen esa característica común. También, si cierto ODS comparte un Principio Cooperativo, los ODS se pueden establecer en una red convencional (modo 1) por esa relación. Así, las matrices que expresan las simples relaciones de modo 1 son matrices booleanas, cuyos elementos son 0 , si no hay relación entre nodos, 0 1, si hay relación. Ahora bien, estas matrices también pueden estar ponderadas o valoradas por la intensidad de la relación.

La expresión visual del grafo se configura sobre el concepto de centralidad. Así, si un nodo tiene más relaciones con el resto y son más intensas, será más central; es decir, más importante en la red. La centralidad del nodo puede ubicarse en el grafo que representa la red bajo algoritmos disponibles en programas como UCINET 6 (Borgatti, et al., 2002) y Netdraw (Borgatti, 2002). Así, a través de un grafo no dirigido (un nodo es causa o consecuencia de otro) o bidireccional (hay una relación biunívoca o relación sin dirección) se intuye la transversalidad de Principios Cooperativos y los ODS (o sobre ellos).

En este trabajo, un conjunto de expertos sobre cooperativismo procedentes de CIRIEC-Colombia valoran la contribución del cooperativismo a los ODS en una matriz de modo 2 bajo una escala Likert 1-3 (DelphiCO, en adelante), de la que es posible obtener las dos redes de modo 1 resultantes sobre la transversalidad de Principios Cooperativos a través de ODS comunes, o de ODS bajo Principios Cooperativos comunes.

En consecuencia, una primera aproximación sobre la correspondencia entre los 17 ODS y los 7 Principios Cooperativos ${ }^{4}$ se puede basar en la teoría de redes y grafos. Por ello, el método desglosa las metas de cada uno de los 17 ODS y aplica una escala a la aportación de los Principios Cooperativos hacia ellos: 1. Difícil, 2. Marginal, 3. Suficiente. Después se obtiene una media de la escala de las metas que componen cada ODS. Paralelamente, se cruza la relación de los Principios Cooperativos y los ODS, de manera que las correspondencias ofrecen una red valorada por la intensidad de la contribución entre los nodos (Principios y Objetivos) implicados.

Conviene señalar que si bien el método Delphi (Adler y Ziglio, 1996) está bien considerado por su versatilidad en diversas áreas de conocimiento, tiende a utilizarse en prospectivas sobre humanidades y ciencias sociales y sobre el avance tecnológico futuro, sea micro o macro (Scott, 2001). Además, es conocido como una técnica basada en un panel de expertos y en la prospección para obtener informa-

4.- Algunos estudiosos del asunto consideran un octavo principio, extendido de la importancia por sí mismo, denominado Principio de Igualdad, junto a los considerados a partir de 1995 por la ACl. 
ción esencialmente cualitativa, pero relativamente precisa, acerca de una realidad focalizada (aunque también puede tratar temas genéricos, especialmente relativos a perspectiva futuras). Por este motivo, se admite que el juicio de los expertos (tanto de CIRIEC-Colombia como extraídos del conjunto de actores de la práctica corriente de la cooperativa colombiana) pueda estar aunada por la quincena de ellos, dado que se recomiendan entre 15 y 30 reflexiones para un asunto concreto, debido a que la preocupación de este trabajo no es sobre un tema general ni global como es la configuración de las interrelaciones entre ODS y Principios Cooperativos.

De lo contrario, se requeriría de un número mucho mayor de expertos y mayor coste. Y téngase en cuenta que de los elementos fundamentales del método destacan el anonimato de los participantes y la respuesta del grupo en forma estadística, como demuestran las encuestas DelphiCO y SondeCO que se imbrican en este trabajo. Así, se siguen los criterios oportunos, como en Landeta (1999) y Campos-Climet et al. (2014), de manera que cada uno de los participantes representa, desde distintos espacios de acción, referentes del cooperativismo colombiano y entre el grupo se destacan algunos de los autores más citados y prolíficos de la investigación en economía solidaria colombiana (Cueto et al., 2018).

Así las cosas, se deduce que los Principios Cooperativos pueden caracterizarse como específicos, con una repercusión mayoritariamente interna, como son los principios de Adhesión Libre, Gestión Democrática y Autonomía, y de entorno amplio, que son los restantes. Estos últimos principios, como los principios de Educación, Servicio a la Comunidad y Cooperación entre Cooperativas, entre otros, son protagonistas de una aportación adicional fuera del entorno específico cooperativo, entendiéndose como externalidades adicionales, debido a que muchos ODS se descomponen en metas a las que, casi exclusivamente, se puede contribuir a través de políticas públicas, y no se abren fácilmente a las contribuciones de otras instituciones componentes del sistema, como las cooperativas. Bien es cierto que, aunque desde los Principios Cooperativos se deducen resultados más específicos hacia la cooperativa (implícitos), también pueden generar, potencialmente, externalidades al entorno general que las rodea.

Por tanto, los grafos representan los nodos más centrales, más influyentes, y con los cuales otros tienen las relaciones más fuertes, así como también presentan una partición Centro-Periferia sobre la mayor densidad de relaciones (y más fuertes) entre ambos grupos (Principios Cooperativos y ODS). Esto ayuda a caracterizar cómo contribuyen, de una manera más intensa o más débil, los Principios Cooperativos a los ODS (Ilustración 2). 


\section{llustración 2. Matriz Centro-Periferia de relaciones entre Principios Cooperativos y ODS}

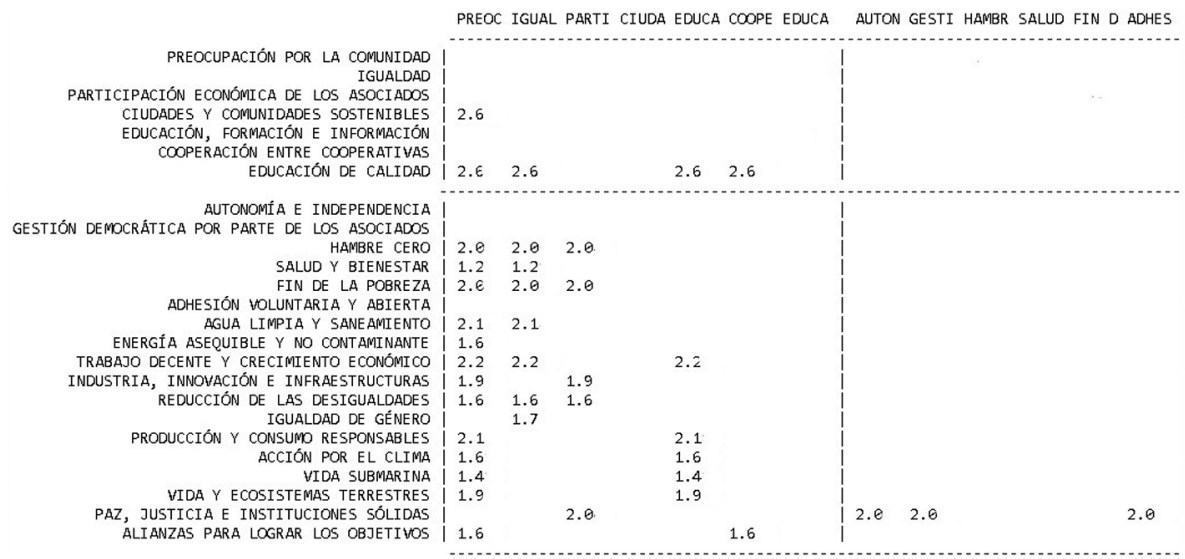

FUENTE: Elaboración propia sobre encuesta DelphiCO y Borgatti, et al. (2002).

El algoritmo "hill climbing" (Russell y Norvig, 2003) en UCINET 6 (Borgatti, 2002) ajusta la partición ideal de los nodos usando el número de relaciones para datos binarios en una partición inicial y un vector propio para los datos valorados que se disponen para las materias, junto con varias particiones aleatorias. El algoritmo itera para ajustar las particiones iniciales de los nodos e informa el mejor ajuste. La función de ajuste es la correlación entre la matriz de nodos permutada y una matriz de estructura ideal que consta de unos en las interacciones del bloque central/nuclear y ceros en las interacciones del bloque periférico.

En consecuencia, la red de modo 2 de Principios cooperativos-ODS agrupa en el núcleo a los principios Participación Económica de los Asociados, Igualdad, Educación, Formación e Información, Cooperación entre Cooperativas y Preocupación por la Comunidad, junto a los ODS Ciudades y Comunidades Sostenibles y Educación de Calidad. El resto de las relaciones muestran menor intensidad, con algunas que casi alcanzan las del núcleo, pero el algoritmo debe particionar la matriz en la categoría que haga manifiesta la falta de similitud, como se observa en los valores de intensidad de relación, las cuales tienden más a aportar externalidades positivas desde los Principios a los ODS.

La llustración 3 destaca la representación de la centralidad, del "poder", de Principios cooperativos y ODS en la red, que viene a corresponderse los nodos más centrales, y destaca el principio de Preocupación por la Comunidad, por ser el nodo de mayor concentración a la contribución de los ODS. En segundo lugar, destaca el principio de Educación, Formación e Información, así sucesiva- 
mente hasta llegar a los Principios más periféricos, pero no por ello poco importante, respecto a su relación con los ODS, como es Autonomía e Independencia que, si bien es relevante para el quehacer cooperativo, destaca por su capacidad de aporte al cumplimiento de algún ODS y disminuye ostensiblemente en su relación a los primeros.

Con lo anterior se quiere señalar que algunos principios tienen una potencialidad importante para que, a partir de las actividades que desarrollan las cooperativas, se procure el cumplimiento de otros de sus Principios cooperativos y, a la vez, que contribuyan a los ODS. Así, se identifican nodos centrales donde se pueden focalizar esfuerzos con el fin de optimizar y medir una contribución más específica de las cooperativas, sea a través de la confección de Balances Sociales, en dos dimensiones: unos más genéricos, que sirvan como referencia comparativa, otros específicos, para la comparativa de una institución cooperativa sea posible respecto al conjunto cooperativo (o por sectores), a los ODS. Medidas robustas de tendencia central u otras serían posibles para poder realizar las comparaciones.

\section{llustración 3. Red de relaciones entre los Principios Coope- rativos (cuadrados rojos) a los ODS (círculos azules)}

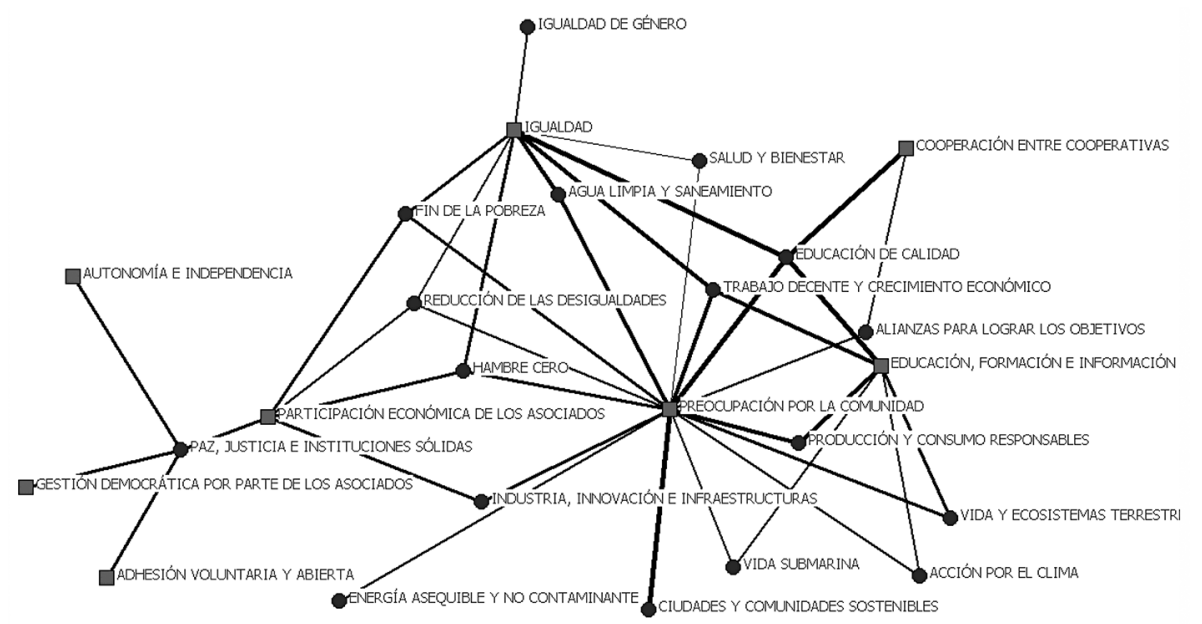

FUENTE: Elaboración propia sobre encuesta DelphiCO, Borgatti (2002) y Borgatti, et al. (2002).

La llustración 4 destaca la representación de los Principios Cooperativos de Preocupación por la Comunidad, y de Igualdad ${ }^{5}$ como ejes transversales del resto del resto de Principios cooperativos y de un buen conjunto de ODS. Es lo que se deduce al aplicar el análisis de componentes principales a 
la matriz de relaciones Principios Cooperativos-ODS. La técnica utilizada en Netdraw (Borgatti, 2002) dibuja el conjunto de datos en términos de nuevas variables, componentes, no correlacionadas. Estos se ordenan por la cantidad de varianza original que describen, por lo que la técnica reduce las dimensiones de un conjunto de datos. Se busca, así, la proyección para la cual los datos queden mejor representados en términos de mínimos cuadrados. En la red basada en este criterio se observa la influencia citada de aquellos Principios Cooperativos dentro del conjunto de díadas posibles entre las dos dimensiones relacionadas que se han considerado.

\section{llustración 4. Red de relaciones entre los Principios Coope- rativos (cuadrados negros) a los ODS (círculos rojos), bajo el criterio de Componentes Principales}

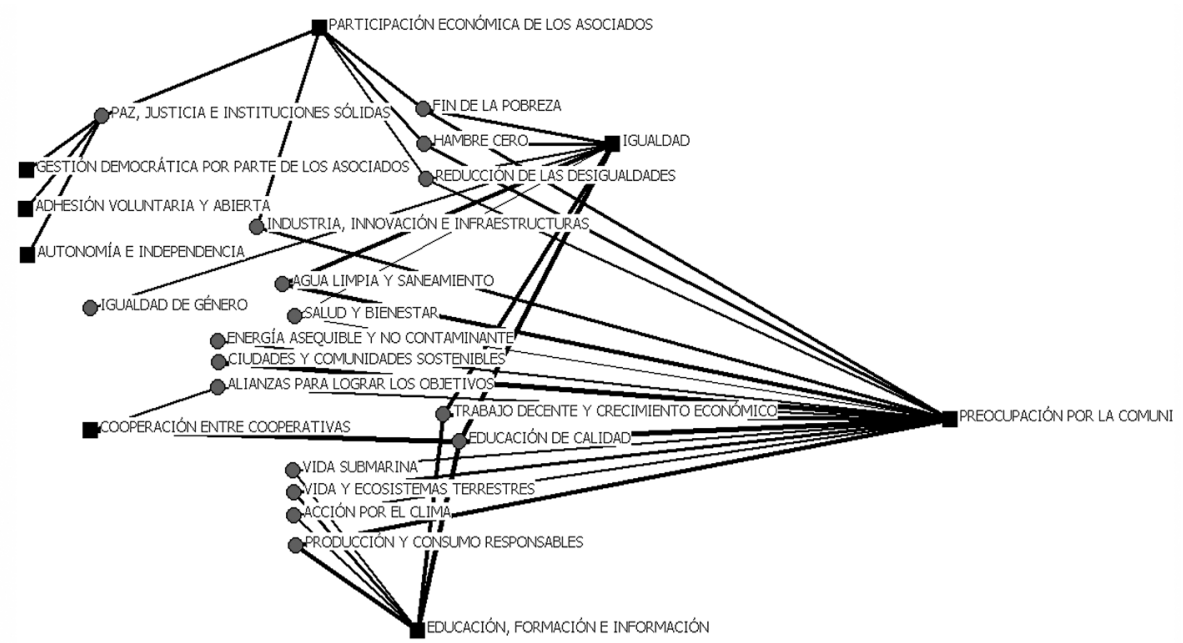

FUENTE: Elaboración propia sobre encuesta DelphiCO, Borgatti (2002) y Borgatti, et al. (2002).

Por otra parte, puede realizarse una agrupación en clústeres de los Principios cooperativos-ODS, que ofrezcan cierta taxonomía de las posibles relaciones entre los componentes de las dos dimensiones (principios-objetivos). En efecto, el agrupamiento es otra técnica del análisis de la interdependencia (como el de Componentes Principales.) En este caso, el procedimiento agrupa una serie de vectores de acuerdo con un criterio, basado en la distancia o la similitud. La cercanía se define en términos de una determinada función de distancia, como la euclídea, y se permite su extensión a variables discretas, como es el caso. La medida más utilizada para medir la similitud entre los casos (díadas, en esta aplicación) es la matriz de correlación. Pertenecer al mismo clúster viene a señalar que se comparten propiedades. En el caso que ocupa aquí, se refiere a las relaciones entre principios 
y objetivos señalados. Así, se obtienen 4 agrupaciones (llustración 5) que ofrecen una taxonomía marcada por:

1. Transversalidad fuerte. Esta agrupación constituye las relaciones de transversalidad y las interdependencias más fuertes entre Principios Cooperativos y ODS. Se compone de principios y objetivos de: Participación Económica de los Asociados; Educación, Formación e Información; Preocupación por la Comunidad; Igualdad; Fin de la Pobreza; Hambre Cero; Educación de Calidad; Agua Limpia y Saneamiento; Trabajo Decente y Crecimiento Económico; Industria, Innovación e Infraestructuras; Reducción de las Desigualdades; Producción y Consumo Responsables; Vida y Ecosistemas Terrestres.

2. Tranversalidad colateral general. Esta agrupación de relaciones se configura sobre externalidades positivas del cooperativismo hacia el entorno más general. Se compone de principios y objetivos de: Cooperación entre Cooperativas; Salud y Bienestar; Vida Submarina; Alianzas para lograr los Objetivos.

3. Trasversalidad colateral específica. Este clúster se configura sobre externalidades positivas del cooperativismo hacia su entorno más específico. Se compone de principios y objetivos de: Adhesión Voluntaria y Abierta; Gestión Democrática por Parte de los Asociados; Autonomía e Independencia; Paz, Justicia e Instituciones Sólidas.

4. Trasversalidad implícita. Esta agrupación de relaciones supone potenciales efectos de internalización hacia el cooperativismo como institución. Se compone de principios y objetivos de: Igualdad de Género; Energía Asequible y No Contaminante; Ciudades y Comunidades Sostenibles, Acción por el Clima. 


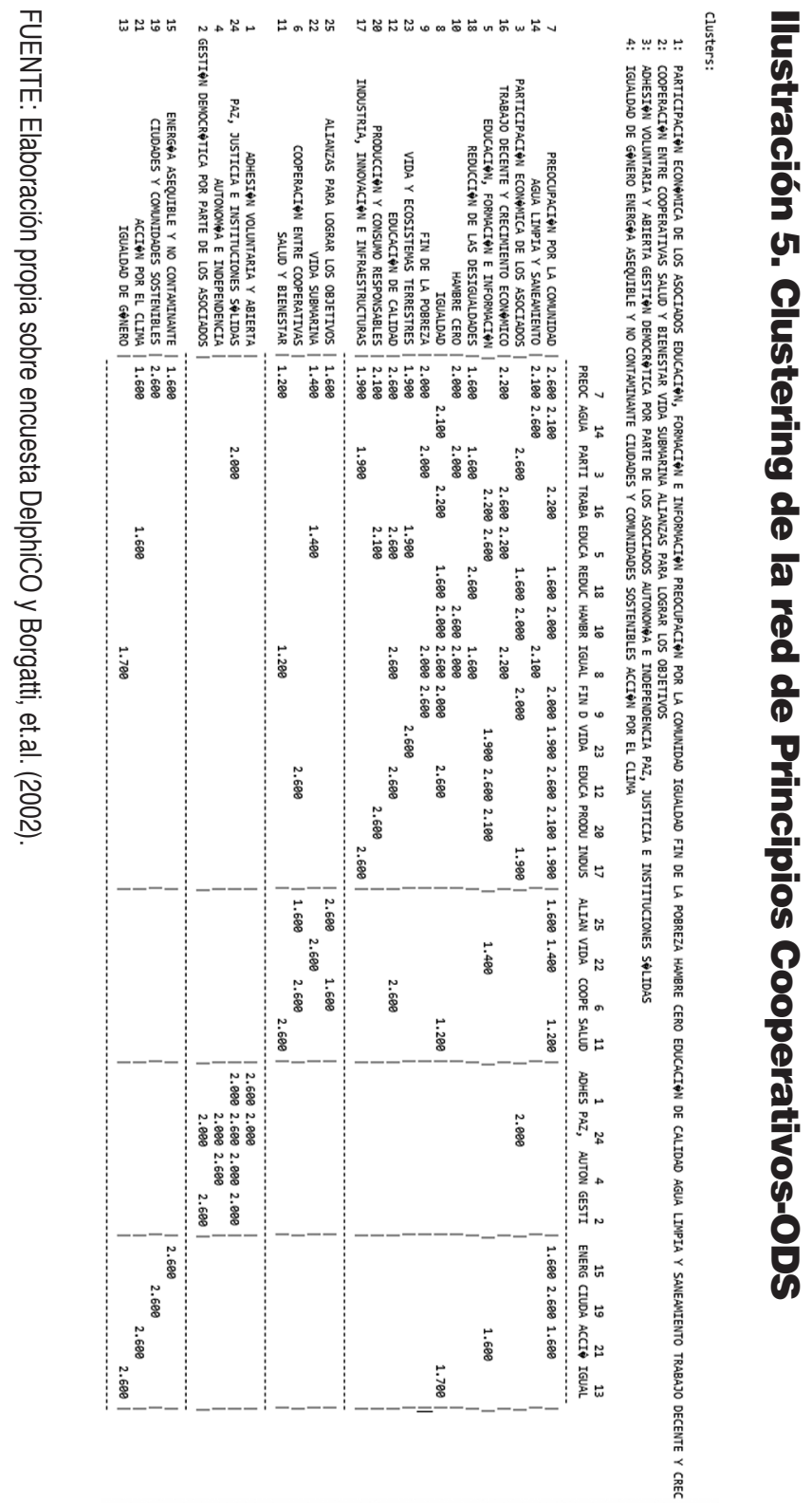




\section{Unos resultados preliminares para cooperativas colombianas}

Partiendo de la sistematización de ciertos cuadros que desarrollan una propuesta de relación entre los ejes del Plan 2020, los Objetivos de Desarrollo del Milenio (ODM) y los Principios Cooperativos, se buscó dar el salto hacia su correspondencia a la Agenda 2030 y los ODS a través de un estudio preliminar en 16 cooperativas de diversos sectores del cooperativismo colombiano a través de un cuestionario con 16 preguntas que se desprenden de los cuadros mencionados, contestado entre septiembre de 2018 y enero de 2019 (SondeoCO, en lo sucesivo).

Los cuadros diseñados relacionaban Variables, Prácticas, Producto esperado, Indicadores, Medios de verificación, Preguntas para depuración, Preguntas para SondeoCO y ODS asociados, para cinco dimensiones: Participación, Sostenibilidad, Identidad, Marco Jurídico y Capital (ver Anexo con un extracto para esta última dimensión). Por último, se eliminaron las dimensiones y se añade una nueva relación con preguntas para el sondeo (Tabla 1).

Las 16 cooperativas seleccionadas acogen empresas de preponderancia sectorial en tanto representaban a los sectores de penetración cooperativa tradicionales y los emergentes. Las cooperativas encuestadas provienen de: a) la multiplicidad de cooperativas financieras del país (7 en total incluyendo cooperativas de aporte y crédito de trabajadores médicos o del sector educativo, empresas solidarias cerradas a trabajadores de una misma empresa, cooperativas especializadas sólo ahorro y crédito y multiactivas con actividad de crédito); b) tres cooperativas de producción (lecheras y trabajo asociado); c) dos organizaciones gremiales cooperativas (el máximo gremio de representación de las cooperativas en el país (Confecoop) y un gremio cooperativo regional); d) una cooperativa tecnológica y especializada en monedas virtuales; e) una cooperativa del tradicional sector de transportadores; f) dos cooperativas promotoras de proyectos ambientales e industriales. Este tipo de cooperativas representan más del $85 \%$ del universo organizacional de las cooperativas en el país, cerca del $90 \%$ de las dimensiones financieras y el $50 \%$ de la generación de empleo.

Si bien el análisis de las respuestas de las cooperativas no es generalizable, debido a que se trata de una muestra no probabilística, es posible dar una aproximación por la importancia cuantitativa de las cooperativas que han respondido. De manera que se vayan consolidando elementos de discusión para próximos acercamientos teóricos y aplicados que se han presentado. La intencionalidad, en todo caso, era (y es) tener propuestas para hacer prácticos los reportes de información en Balance Social con el objetivo de que se termine agregando datos en materia de ODS y Agenda 2030 (Tabla 1). 


\section{Tabla 1. Resultados preliminares sobre relaciones ODS-Principios Cooperativos en el entomo Agenda 2030 en Colombia, 2019}

\begin{tabular}{|c|c|}
\hline & Media \\
\hline $\begin{array}{c}\text { ¿Qué porcentaje aproximado de asociados son mujeres y cuántas de ellas } \\
\text { participan en las iniciativas de la cooperativa? }\end{array}$ & $44,8 \%$ \\
\hline ¿Cuántas de ellas participan en las iniciativas de la cooperativa? & $29,5 \%$ \\
\hline $\begin{array}{l}\text { ¿Cuánto porcentaje de los asociados son menores de } 30 \text { años? y ¿cuántos } \\
\text { participan en las iniciativas de la cooperativa? }\end{array}$ & $9,4 \%$ \\
\hline ¿A qué Objetivos de Desarrollo Sostenible contribuye su cooperativa? & $\begin{array}{l}\text { 4/5 señalan en común Ciudades y Comunidades } \\
\text { Sostenibles y Educación de Calidad }\end{array}$ \\
\hline $\begin{array}{l}\text { ¿Qué actividades desarrolló para el cumplimiento cada uno de los Objetivos } \\
\text { de Desarrollo Sostenible? }\end{array}$ & $\begin{array}{l}\text { 2/3 señalan actividades relacionadas con Princi- } \\
\text { pios Cooperativos de Participación Económica de } \\
\text { los Asociados, Igualdad, Educación, Formación } \\
\text { e Información, Cooperación entre Cooperativas y } \\
\text { Preocupación por la Comunidad }\end{array}$ \\
\hline ¿Qué porcentaje de sus excedentes invierte en ODS? & $23,5 \%$ \\
\hline $\begin{array}{l}\text { ¿Posee su cooperativa, u otras, una posición de dominio en su sector de } \\
\text { mercado? }\end{array}$ & $6,3 \%$ \\
\hline ¿Su cooperativa presenta Balances Sociales? & $37,5 \%$ \\
\hline $\begin{array}{l}\text { ¿La actividad de la cooperativa está relacionada con el territorio y la } \\
\text { generación de empleo? }\end{array}$ & $68,8 \%$ \\
\hline ¿Cómo genera su cooperativa valor social y ambiental? & $\begin{array}{l}\text { Similares en puntos porcentuales sobre referen- } \\
\text { cias de mercado, a través de precios e intereses, } \\
\text { programas sociales y a través de la educación. }\end{array}$ \\
\hline $\begin{array}{l}\text { Si su cooperativa está relacionada al ahorro y al crédito ¿cuál es el diferen- } \\
\text { cial aproximado, en puntos porcentuales, entre los intereses del mercado y } \\
\text { los de su cooperativa? }\end{array}$ & $4-10$ puntos $\%$ \\
\hline $\begin{array}{l}\text { ¿Cuál es el diferencial, sea positivo o negativo, en puntos porcentuales de } \\
\text { las compensaciones salariales pagados por la cooperativa en relación al } \\
\text { mercado? }\end{array}$ & $6-9$ puntos $\%$ \\
\hline $\begin{array}{l}\text { ¿Cuál es el diferencial porcentual en precios de los productos o servicios } \\
\text { que oferta en los mercados? }\end{array}$ & $10-15$ puntos $\%$ \\
\hline $\begin{array}{l}\text { ¿Incorpora tecnología de punta, tecnologías propias o tradicionales en su } \\
\qquad \text { actividad? }\end{array}$ & $62,5 \%$ \\
\hline ¿Envía informes de gestión a sus asociados? Si no, ¿lo considera? & $68,8 \%$ \\
\hline ¿Su cooperativa ofrece productos o servicios sostenibles? & $\begin{array}{l}\text { La sostenibilidad está asociada para las Coopera- } \\
\text { tivas de Educación como eje transversal. }\end{array}$ \\
\hline ¿Su cooperativa realiza o financia actividades sostenibles? & $81,3 \%$ \\
\hline
\end{tabular}

FUENTE: Elaboración propia sobre SondeoCO-2019. 
De forma que el análisis genérico de las respuestas responde con interesantes registros sobre indicadores relativos de tipo cualitativo y cuantitativos (Tabla 1):

- En la cuestión de género, se puede hablar de paridad, pero a favor de los hombres en unos pequeños puntos porcentuales. En Consejos de administración y cuerpos directivos la tendencia central es que $1 / 3$ de las mujeres ocupen estos cargos.

- Respecto al porcentaje de asociados jóvenes, sólo 1 de cada 10 son miembros de las cooperativas, más su participación es mucho menor. Ello da cuenta de la poca juventud de los miembros de las cooperativas y es posible que genere un techo de cristal para el crecimiento organizacional.

- Se constata que la mayoría de las organizaciones están en sintonía con los nodos centrales de las redes (que no son otra cosa que los ODS con mayor correspondencia con los principios cooperativas presentados en la llustración 3).

- Llama la atención que las cooperativas destinan cerca de 1/4 de sus excedentes en acciones que contribuyen a los ODS, fundamentalmente porque muchas de las finalidades cooperativas son externalidades y terminan repercutiendo en los mismos. Ello comporta el reto de valorar las externalidades e internalizar su cálculo dentro del Balance Social, que por supuesto será más alto que el que se intuye hasta este momento. Lo que reafirma la sintonía entre los principios cooperativistas y los ODS, y la utilidad del Balance Social como instrumento de valoración de los aportes cooperativistas a los ODS.

- Esto se ve correspondido con el desarrollo de actividades implementados por las cooperativas, especialmente en materia de preservación medioambiental, captura carbono, trabajo decente y educación. Las intenciones se convierten, en consecuencia, en acciones concretas para las cuales se identifican los aportes. Pero será menester calcularlos a partir de la internalización de esas externalidades en el Balance Social.

- Las cooperativas no poseen posiciones de dominio en el mercado, pero si son muy importantes dado que son referentes en su contexto y contribuyen a mejorar las orientaciones al bien común en los mercados. Es el caso de cooperativas en los sectores médico, crediticio, de transporte, editorial, lechero, e incluso en zonas aisladas donde terminan siendo experiencias para quienes tienen espacios de mercado para desarrollar un accionar responsable.

- En general la mayoría de las cooperativas pretende presentar Balance Social lo cual es un símbolo de transparencia, Sin embargo, no hay metodologías estándar para reportar.

- Sí que se han efectuado acciones en pro de los ODS, pero se quedan a nivel de comunicación con sus asociados y no tienden a reportarse lo cual coincide con los hallazgos de (Álvarez, Zabala y Orrego, 2016).

- Las cooperativas realizan acciones en su territorio. 2 de cada 3 organizaciones inciden en él, pero reconocen que les falta documentar, articular y proyectar sus acciones con la comunidad.

- Las cooperativas generan valor en porcentajes excedentes (sociales) similares a través de precios e intereses (Ballestero, 1984), programas sociales y a través de la educación (Álvarez \& Blanco, 2014), coincidente con las teorías de generación de valor cooperativo. 
- La diferencia entre los precios de mercados y los de cooperativas están entre 5 y 10 puntos porcentuales; en compensaciones es cercano a los 10 puntos porcentuales a favor de los trabajadores de cooperativas quienes además reciben compensaciones integrales que se concentran en las empresas de capital casi exclusivamente a cargos gerenciales; respecto al diferencial en la oferta de servicios, es de casi del 10 a 15 puntos porcentuales a favor de los asociados.

- Poco más de $1 / 3$ de las cooperativas no incorporan tecnología.

- La sostenibilidad está asociada para las cooperativas a la educación como eje transversal.

- La gran mayoría de cooperativas, más de un 80 por 100, realiza o financia iniciativas sostenibles.

\section{Conclusiones}

Muchos de los aportes sustantivos que realizan las empresas de la ESS y, por tanto, las cooperativas no pueden ser obtenidos bajo la óptica de la economía tradicional. Por ello, la percepción de la incidencia de las cooperativas y sus aportes a los Objetivos de Desarrollo Sostenible requieren alternativas y crecientes soportes para demostrarlos. Sin embargo, faltan ejercicios más representativos y agregados que apunten a generar conclusiones contundentes sobre el tema y que hagan uso de diferentes medios de verificación con el fin de ganar confiabilidad empírica. Los ejercicios realizados aquí son una aproximación susceptible de debates y mejoras y a su vez abiertas a su réplica en diferentes contextos.

Tras el Análisis de Redes efectuado, es posible derivar una relación estrecha entre los Principios Cooperativos y las metas de los Objetivos de Desarrollo Sostenible, y resulta que para el caso colombiano hay evidencias que señalan que las cooperativas contribuyen al cumplimiento de los ODS en atención a la puesta en valor de sus principios. El cuestionario realizado a 16 cooperativas de Colombia (SondeCO) muestra, por ejemplo, que, en promedio, las cooperativas generan entre 10 y 15 puntos porcentuales de TSE (ampliadas) hacia colectivos que abarcan diversos stakeholders.

Los hallazgos encontrados enfatizan que principios como el interés por la comunidad y la educación, formación e información tienen mayor relación con el cumplimiento de metas de los ODS y por tanto las prácticas que se realizan en atención a estos principios tenderán a generar más contribuciones transversales. Pero ello no menoscaba la importancia de los otros Principios Cooperativos en tanto forman parte de un ecosistema organizacional de múltiples actores que hacen parte de las cooperativas. Con ello, los hallazgos refuerzan a la identidad cooperativa como un elemento central para direccionar las contribuciones de las cooperativas a los acuerdos de la Agenda 2030. 
Por otra parte, el análisis de los hallazgos y su relación con la teoría previa de Balance Social permite encontrar vacíos en el reporte del accionar cooperativo que conviene llenar. Para abordar la dimensión plural de las cooperativas se requiere identificar y valorar los bienes de mercado y de no mercado, monetarios y no monetarios resultantes de su actividad. A nivel microeconómico, esas transacciones pueden ser reportadas en un Balance Social, establecer distintos niveles meso-económicos y poder, así, conectar rúbricas seleccionadas a nivel macro a través de unas cuentas satélite de la economía social. Un aspecto nuclear es el estudio tratado de las transferencias sociales en especie y de la extensión de su concepto actual en los Sistemas de Cuentas Nacionales (TSE ampliadas).

De hecho, del razonamiento sobre el uso de múltiples opciones fuera del entorno específico de este trabajo, referido al tipo de entidad y escuela de pensamiento, subyace que no es fácil ni factible, por ahora, un consenso en indicadores micro, meso y macroeconómicos de manera integral. Por tanto, quedan ingentes retos como trabajar en herramientas que permitan la agregación entre Balances Sociales individuales, propiciar criterios para valorar la liberación de recursos generados por los diferenciales que obtienen los cooperativistas por sus transacciones con su cooperativa en relación a los precios del mercado, cuantificar los excedentes sociales entendidos como aquellos bienes y servicios que obtienen los cooperativistas por las acciones no monetarias y valorar los bienes preferentes 0 meritorios.

Con lo anterior, podemos concluir que para optimizar el reporte de los aportes de las cooperativas a las metas de los ODS debería ser posible apelar a la agregación de datos presentados en el Balance Social y a herramientas de agregación meso-económica. Después, que ambos se engarcen con ciertos indicadores "estrella" en la dimensión macroeconómica, que resuman el circuito económico y social de la actividad de las entidades de la ESS, particularmente el de las cooperativas, como una suerte de cuentas satélite. Y convendría que se centraran especialmente en el cálculo de las magnitudes de las cuentas de Distribución de la Renta Disponible y de la Renta Disponible Ajustada, que son las que se centran en el tratamiento de las TSE.

Estos abordajes conceptuales y aplicados están en sintonía con el enfoque del institucionalismo económico contemporáneo, porque al estudiar las cooperativas la propuesta concentra su atención en los esfuerzos que realizan las empresas en la generación de beneficios compartidos que finalmente contribuyen al bien común y por supuesto a los ODS, a través del hábito de la No Prioridad en el Ánimo de Lucro Personal, y realizan rutinas específicas emitiendo TSE.

Todo lo anterior, con las cautelas a tener en cuenta respecto a los sondeos practicados y la generalización de conclusiones, solo válidas en el entorno cultural, territorial y sectorial tratado, y para la propuesta teórica señalada. 


\begin{tabular}{|c|c|c|c|c|}
\hline 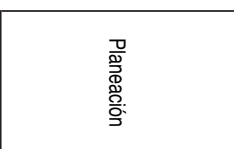 & 옹: & 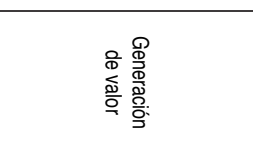 & 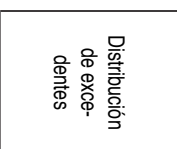 & 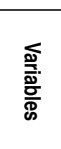 \\
\hline 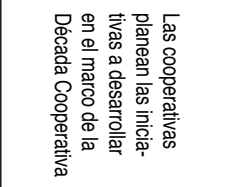 & 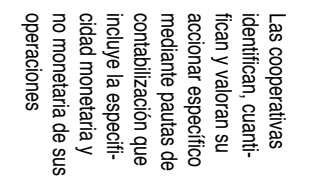 & 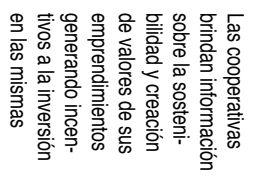 & 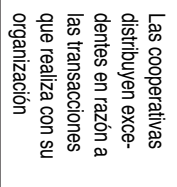 & 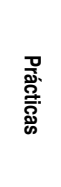 \\
\hline 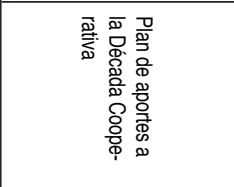 & 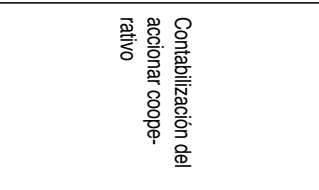 & 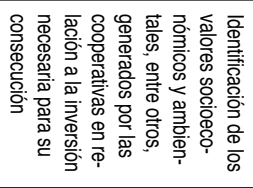 & 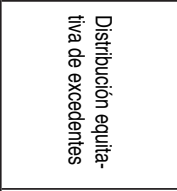 & 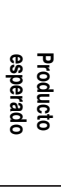 \\
\hline 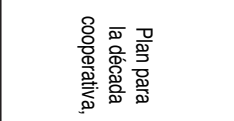 & 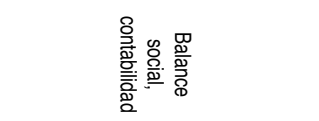 & 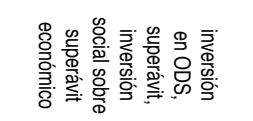 & 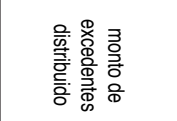 & 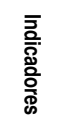 \\
\hline 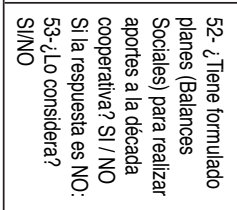 & 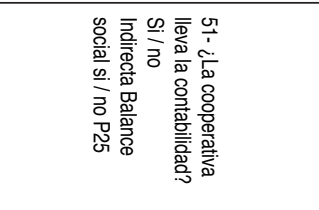 & 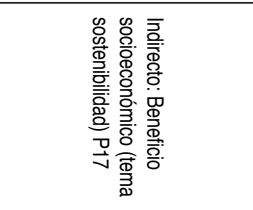 & 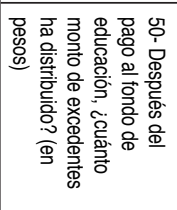 & 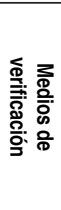 \\
\hline 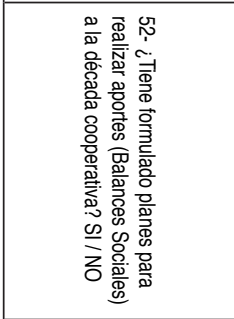 & 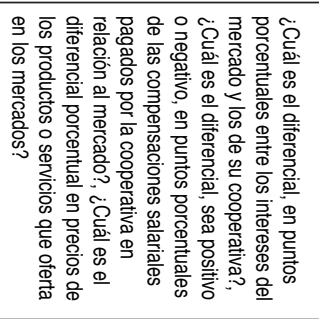 & 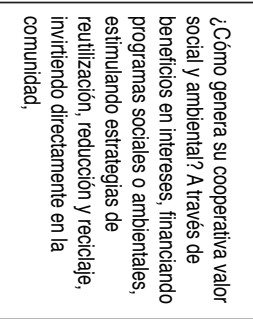 & 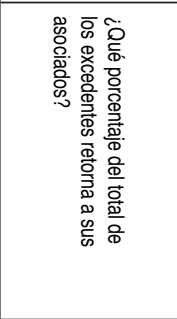 & 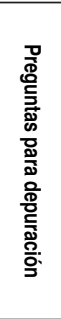 \\
\hline 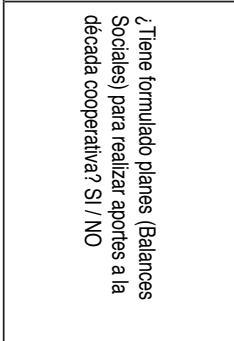 & 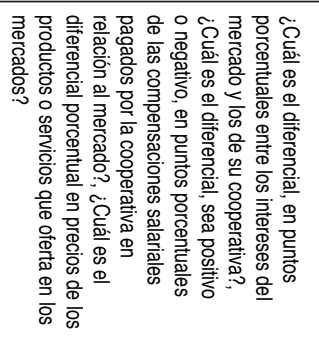 & 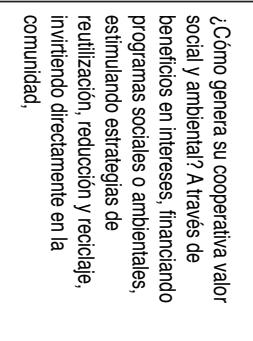 & 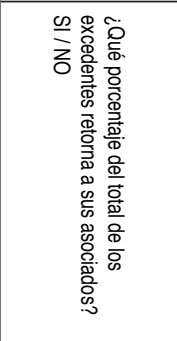 & 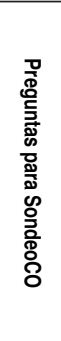 \\
\hline 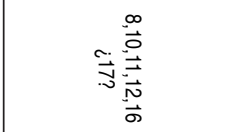 & $\begin{array}{l}\infty \\
\stackrel{\infty}{0} \\
\doteq \\
\overrightarrow{\vec{N}} \\
\vec{\sigma}\end{array}$ & $\vec{\nabla} \underset{\overrightarrow{0}}{\stackrel{\overrightarrow{0}}{\vec{\sigma}}}$ & $\begin{array}{l}\overrightarrow{0} \\
\stackrel{\vec{\Xi}}{\vec{N}} \\
\vec{\sigma}\end{array}$ & 옹 \\
\hline
\end{tabular}




\section{Bibliografía}

ADLER, M. \& ZIGLIO, E. (1996): Gazing Into the Oracle: The Delphi Method and Its Application to Social Policy and Public Health, Jessica Kingsley Publishers, Londres-Filadelfia.

ALARCÓN, M.A. (2016): "La economía social desde el institucionalismo económico. Evidencia empírica", CIRIEC-España, Revista de Economía Pública, Social y Cooperativa, 86, 61-100. DOI: 10.7203/CIRIEC-E.86.8167.

ALARCÓN, M.A. y ÁLVAREZ, J.F. (2018): "La dimensión sectorial del cooperativismo colombiano a través de un análisis de redes", CIRIEC-España, Revista de Economía Pública, Social y Cooperativa, 92, 121-154. DOI: 10.7203/CIRIEC-E.92.10606.

ALARCÓN, M.A. (2019): "The Measurement of the Social and Economic Value on The Social Economy from a Modern Institutional Economics Approach". Comunicación en 7th Ciriec International Research Conference on Social Economy (Bucharest, Romania), June 6-9, 2019 (http://www. ciriec.uliege.be/wp-content/uploads/2019/06/E2-MEASUREMENT-OF-THE-SOCIAL-AND-ECONOMIC-VALUE.pdf)

ALIANZA COOPERATIVA INTERNACIONAL (2013): Plan para una Década Cooperativa, Bruselas.

ÁLVAREZ, J.F. (2017): Economía social y solidaria en el territorio: significantes y co-construcción de políticas públicas. Bogotá: Colección Escuela Javeriana de Gobierno y Ética Pública No.2. Pontificia Universidad Javeriana. Bogotá.

ÁLVAREZ, J.F. y BLANCO, L. (2014): "Balance social y valoración de los recursos liberados en las cooperativas". En: Tendencias y temas que inciden en el desarrollo de las disciplinas ejes de la Contaduría Pública, Universidad de Guadalajara, Guadalajara-México.

ÁLVAREZ, J.F., ZABALA, H. y ORREGO, C. (2016): Diagnósticos y recomendaciones en torno a los emprendimientos cooperativos colombianos dispuestos para el cumplimiento del Plan 2020, Contrato 207-052-2016, IEMP-Procuraduría General de la Nación, Bogotá.

ARGANDOÑA, A., LOSADA, C. y TORRALBA, F. (2015): Una economía al servicio de la persona, Editorial Milenio, Lleida.

BALLESTERO, E. (1983): Teoría económica de las cooperativas, Alianza Universidad, Madrid.

BASSI, A. \& VINCENTI, G. (2015): "Toward a New Metrics for the Evaluation of the Social Added Value of Social Enterprises", CIRIEC-España, Revista de Economía Pública, Social y Cooperativa, 83, 9-42. DOI: 10.7203/CIRIEC-E.93.9953. 
BORGATTI, S.P. (2002): NetDraw: Graph Visualization Software, Analytic Technologies, Harvard.

BORGATTI, S.P., EVERETT, M.G. \& FREEMAN, L.C. (2002): Ucinet 6 for Windows: Software for Social Network Analysis, Analytic Technologies. Harvard.

BRUNDTLAND, G. (Presidenta de la comisión mundial sobre el medio ambiente y el desarrollo, y sus miembros) (1987): Nuestro futuro común, Naciones Unidas, Nueva York. Disponible en https:// undocs.org/es/A/42/427 2/11/2018).

CAMPOS-CLIMENT, V., MELIÁN, A. y SANCHIS, R. (2014). "El método Delphi como técnica de diagnóstico estratégico. Estudio empírico aplicado a las empresas de inserción en España", Revista Europea de Dirección y Economía de la Empresa, 23(2), 72-81. DOI:10.1016/j.redee.2013.06.002.

CASTILLA, F. y GALLARDO, D. (2014): "La revelación social en sociedades cooperativas: una visión comparativa", REVESCO, Revista de Estudios Cooperativos, 114, 7-34. DOI: 10.5209/rev REVE.2014.v114.44291.

CONSEJO ECONÓMICO Y SOCIAL-ESPAÑA (2016): Informe Nuevos hábitos de consumo, cambios sociales y tecnológicos, Sesión ordinaria del Pleno de 23 de noviembre de 2016, Número 04/2016. Madrid.

CRACOGNA, D. (2015): "La legislación cooperativa latinoamericana en el marco del Plan para una Década Cooperativa de la Alianza Cooperativa Internacional", REVESCO, Revista de Estudios Cooperativos, 117, 12-33. DOI: 10.5209/rev REVE.2015.v117.48416.

CUETO, E., ARBOLEDA, O., ZABALA, H. y ECHEVERRY, F. (2018): Una década de economía social y solidaria en Colombia. Análisis de la producción investigativa y académica 2005-2015, UNIMINUTO - Universidad Católica Luis Amigó, Medellín. Disponible en: http://hdl.handle.net/10656/7235.

CHAVES, R. (1999): "La economía social como enfoque metodológico, como objeto de estudio y como disciplina científica", CIRIEC-España, Revista de Economía Pública, Social y Cooperativa, 33, 115-139.

ETXEZARRETA, E., MENDIGUREN, J.C.P., DIAZ, L. y ERRASTI, A. (2018): "Valor social de las cooperativas sociales: aplicación del modelo poliédrico en la cooperativa para la acogida de menores Zabalduz S. Coop", CIRIEC-España, Revista de Economía Pública, Social y Cooperativa, 93, 155-180. DOI: 10.7203/CIRIEC-E.93.9953.

FELBER, C. (2013): La economía del bien común, $6^{\circ}$ edición: Ediciones Deusto, Barcelona.

GARCÍA, A. (2017): Derecho cooperativo, mutual y de la economía social y solidaria, CIRIEC-Colombia. Bogotá.

KELLOGG COLLEGE-ACI (2012): Proyecto de una Década Cooperativa. ACl. Ginebra.

LANDETA, J. (1999): El método Delphi: una técnica de previsión para la incertidumbre, Barcelona: Ariel. 
MARTÍ, J.P. (2016): "La consecución de los objetivos del desarrollo sostenible y el "milagro" cooperativo", Memorias IV Cumbre Cooperativa de las Américas, Montevideo.

MUGARRA, A. (2001): "Responsabilidad y Balance Social hoy en día: un reto para las Cooperativa", CIRIEC-España, Revista de Economía Pública, Social y Cooperativa, 39, 25-50.

NARRILLOS, H. (2012): Economía social: valoración y medición de la inversión social (método SROI), Ediciones Ecobook, Madrid.

OIT-ACI (2014): Las cooperativas y los Objetivos de Desarrollo Sostenible. Debate sobre el desarrollo después de 2015. Informe de política, Ginebra.

ONU (2010): Las cooperativas en el desarrollo social. Resolución A/RES/64/136, Nueva York.

ONU (2014): La Economía Social y Solidaria y el Reto del Desarrollo Sostenible, Ginebra.

ONU (2015): Transformar nuestro mundo: la Agenda 2030 para el Desarrollo Sostenible, Resolución A/RES/70/1. Nueva York.

ONU (2018): Informe de los Objetivos de Desarrollo Sostenible 2018, Nueva York.

RETOLAZA, J.L., SAN-JOSE, L. \& RUIZ-ROQUEÑI, M. (2015): "Monetarizing the social value: theory and evidence", CIRIEC-España, Revista de Economía Pública, Social y Cooperativa, 83, 43-62. DOI: 10.1515/erj-2013-0074.

ROELANTS, B. (2013): Crecimiento cooperativo para el Siglo XXI. Informe de la CICOPA. Extraído el 6 de septiembre de 2016 del sitio http://www.aciamericas.coop/IMG/pdf/crecimiento_cooperativo_para_el_siglo_xxi.pdf.

RUSSELL, S.J. \& NORVIG, P. (2003): Artificial Intelligence: A Modern Approach (2nd ed.), Prentice Hall. Upper Saddle River, New Jersey, 111-114. DOI: 10.1016/j.artint.2011.01.005.

SALATHÉ-BEAULIEU, G., BOUCHARD, M. \& MENDELL, M. (2019): Sustainable Development Impact Indicators for Social and Solidarity Economy. State of the Art, Working Paper 2019-4 UNRISD, United Nations Research Institute for Social Development, Geneva. DOI: 10.13140/ RG.2.2.13897.77923.

SATGAR, V. (2013): "Una respuesta del movimiento cooperativo a la crisis de la civilización: jelegimos la sostenibilidad de la vida!". En: ACI-CICOPA, Crecimiento Cooperativo para el siglo XXI, Coordinado por B. Roelants, Ginebra.

SCOTT, G. (2001): "Strategic Planning for High-Tech Product Development", Technology Analysis \& Strategic Management, 13(3), DOI: 10.1080/09537320120088174.

SOCIAL ECONOMY EUROPE (2013): Measurement and Evaluation of Social Impact, Position Paper Draft 2013-056a, Luxemburgo. 
STIGLITZ, J.E., SEN, A. \& FITOUSSI, J.P. (2009): Measurement of Economic Performance and Social Progress, Commission on the Measurement of Economic Performance and Social Progress. DOI: 10.1787/9789264307292-en https://ec.europa.eu/eurostat/documents/118025/118123/Fitoussi+Commission+report.

UNRISD (2019): Implementing the Sustainable Development Goals: What Role for Social and Solidarity Economy? ,Conference summary. Disponible en: http://www.unrisd.org/80256B3C005BCCF9/ httpNetITFramePDF?ReadForm\&parentunid=09940E13E1EC57B48025848D003080C5\&parentdoctype=brief\&netitpath=80256B3C005BCCF9/(httpAuxPages)/09940E13E1EC57B48025848D003080C5/\$file/Conference-summary---UNTFSSE-Conference-07.10.2019-Final.pdf

UTTING, P. (2018): Achieving the Sustainable Development Goals through Social and Solidarity Economy: Incremental versus Transformative Change. KNOWLEDGE HUB WORKING PAPER, UN Inter-Agency Task Force on Social and Solidarity Economy, UNSRID, Geneva. 
